\title{
Vigilância entomológica da doença de Chagas no Estado de São Paulo*
}

\author{
Dalva Marli Valério Wanderley**
}

\begin{abstract}
WANDERLEY, D. M. V. Vigilância entomológica da doença de Chagas no Estado de São Paulo, Brasil. Rev. Saúde públ., S. Paulo, 25: 28-32, 1991. Modificações no espaço agrário paulista com redução das matas primitivas e alteração no sistema de produção rural com o assalariamento, culminaram com a expulsão do trabalhador do campo para a cidade. Ao lado desta reestruturação, o trabalho intenso e contínuo de intervenção por parte da Superintendência de Controle de Endemias (SUCEN), no controle de triatomíneos domiciliados através da utilização de inseticidas, conduziu à interrupção da transmissão vetorial da endemia chagásica, no início da década de 70, atingindo-se a fase de vigilância epidemiológica. Esta fase avança para a integração do controle vertical do vetor na assistência primária à saúde, envolvendo a participação da população. Vários anos de trabalho junto às populações rurais foram importantes para orientá-las no sentido de entender o significado da presença de triatomíneos em suas casas e notificá-los à SUCEN. O pronto atendimento a cada notificação é importante para estimular o processo. Informações de 1985 e 1986 mostraram que $85,6 \%$ dos triatomíneos capturados no interior das casas foram notificados pelos moradores, mostrando que para a manutenção dos domicílios livres desses insetos é de fundamental importância a participação da população.
\end{abstract}

Descritores: Tripanossomose Sul-Americana, prevalência. Vigilância epidemiológica. Controle de insetos. Participação comunitária.

\section{Introdução}

No Estado de São Paulo, transformações no espaço geográfico com redução das matas primitivas e modificações no sistema de produção rural com o assalariamento, culminaram com a expulsão do trabalhador rural do campo para a cidade ${ }^{8}$. Ao lado da reestruturação do espaço agrário paulista, o trabalho intenso e contínuo de intervenção por parte da Superintendência de Controle de Endemias (SUCEN), no controle aos triatomíneos domiciliados através da utilização de inseticidas, conduziu à interrupção da transmissão vetorial da endemia chagásica, no início da década de 70 9,10.

Após a longa campanha levada a efeito contra o vetor domiciliário da doença de Chagas, Triatoma infestans, conseguiu-se atingir no Estado de São Paulo a denominada fase de vigilância entomológica.

A Região de Bauru, localizada a oeste do Estado, foi pioneira numa experiência envolvendo a comunidade na vigilância triatomínica, conforme descrito por Rocha e Silva e col. ${ }^{7}$ Nessa árca piloto de vigilância, a deteç̧ão de focos de triatomíneos foi delegada a uma rede de postos de notificação, envolvendo escolas rurais e centralizada num órgão

* Trabalho apresentado no Congresso do $50^{\circ}$ Aniversário do Instituto de Medicina Tropical "Pedro Kouri", La Habana, Cuba, 1988.

** Superintendência de Controle de Endemias (SUCEN) Rua Paula Souza, 166 - 01027 — São Paulo, SP - Brasil. coordenador na cidade de Bauru. Reporta-se "grande participação e entusiasmo do professorado, facilidade operacional e aumento do grau de consciência entre a população quanto a doença de Chagas"7. Embora esta experiência tenha sido suspensa, por razões possivelmente administrativas, apesar de ter servido de estímulo para projetos em outras áreas ${ }^{4}$, o esquema de vigilância paulista logrou integrar a participação de moradores no Programa de Controle da Doença de Chagas ${ }^{10}$, porém não da forma proposta por aqueles autores.

Ao longo dos anos a notificação de triatomíneos, por parte da população, sempre foi incentivada pelas equipes de campo que atuam na pesquisa triatomínica de rotina em casas da área rural paulista. Mais recentemente, passou-se a treinar estas equipes para que pudessem fornecer à população informações necessárias à comprecnsão da importância do trabalho de pesquisa de triatomíncos, solicitando, quando possível, a presença do morador no decorrer da mesma, orientando-o quanto aos locais mais habituais de encontro de "barbeiros".

Critćrios de vigilância introduzidos no Estado de São Paulo a partir de $1984^{2,9,10}$, preconizaram pesquisa triatomínica de rotina, casa a casa, na área originalmente considerada endêmica e a priorização de pesquisas em casas de moradores que encaminhassem triatomíneos, numa atividade designada "atendimento à notificação". Este atendimento tem como meta o incentivo à participação da população no Programa, não deixando nenhuma notificação sem resposta. 
Em fase de vigilância entomológica, a participação da população tem-se mostrado de grande eficácia na descoberta de focos de triatomíneos, sobrepujando, muitas vezes, a pesquisa domiciliar de rotina. Em algumas áreas, a população está familiarizada com a eventual domiciliação de triatomíneos; o imediato atendimento à notificação, por parte da SUCEN, através da realização de pesquisa cuidadosa de amplitude do foco e do expurgo do mesmo, quando indicado, constitui uma forma de garantir a credibilidade no Programa e, ao mesmo tempo, assegurar que a área permanece sob controle.

Por outro lado, as notificaçōes constituem seleção prévia das casas a serem pesquisadas e, portanto, com maior chance de serem encontradas infestadas. Em 1983 verificou-se que a pesquisa nas casas onde 0 morador encaminhou triatomíneos para a SUCEN apresentou positividade de $12,8 \%$ contra $0,14 \%$ nos trabalhos de pesquisa de rotina, dados que mostram a eficiência dessa atividade ${ }^{11}$.

No presente trabalho são apresentadas as informações referentes às espécies triatomínicas capturadas no Estado de São Paulo em atividade de rotina e através da notificação de moradores nos dois anos seguintes à introdução dos critérios citados, 1985 e 1986, e sempre que possivel, apontadas algumas medidas que possam ser incorporadas na orientação da vigilância.

\section{Materlal e Método}

As informações coletadas em nivel de campo e laboratório pela SUCEN foram registradas em impressos próprios, processadas e consolidadas em computador e recuperadas em forma de relatório.

Para análise das atividades de pesquisa de campo foram utilizadas informações de infestação de casas (intradomicílios) e peridomicílios segundo as atividades de rotina e notificação.

Em nível de laboratório foram condensadas as informaçōes sobre a identificação de triatomíneos aliados aos índices de infecção natural.

\section{Resultados e Discussáo}

Os triatomíneos de maior presença no Estado de São Paulo atualmente são Triatoma sordida e Pans. trongylus megistus, cujos habitats são freqüentemente invadidos e modificados pelo homem. Em áreas sob alta ação antrópica, os reservatórios silvestres de triatomíneos acabam por esgotar-se, permitindo respostas muito boas à profilaxia. Ao contrário, as espécies nativas e de maior valência ecológica têm-se mostrado, naturalmente, muito mais problemáticas ao controle do que aquelas mais restritas ao intradomicílio. (Fig. 1).

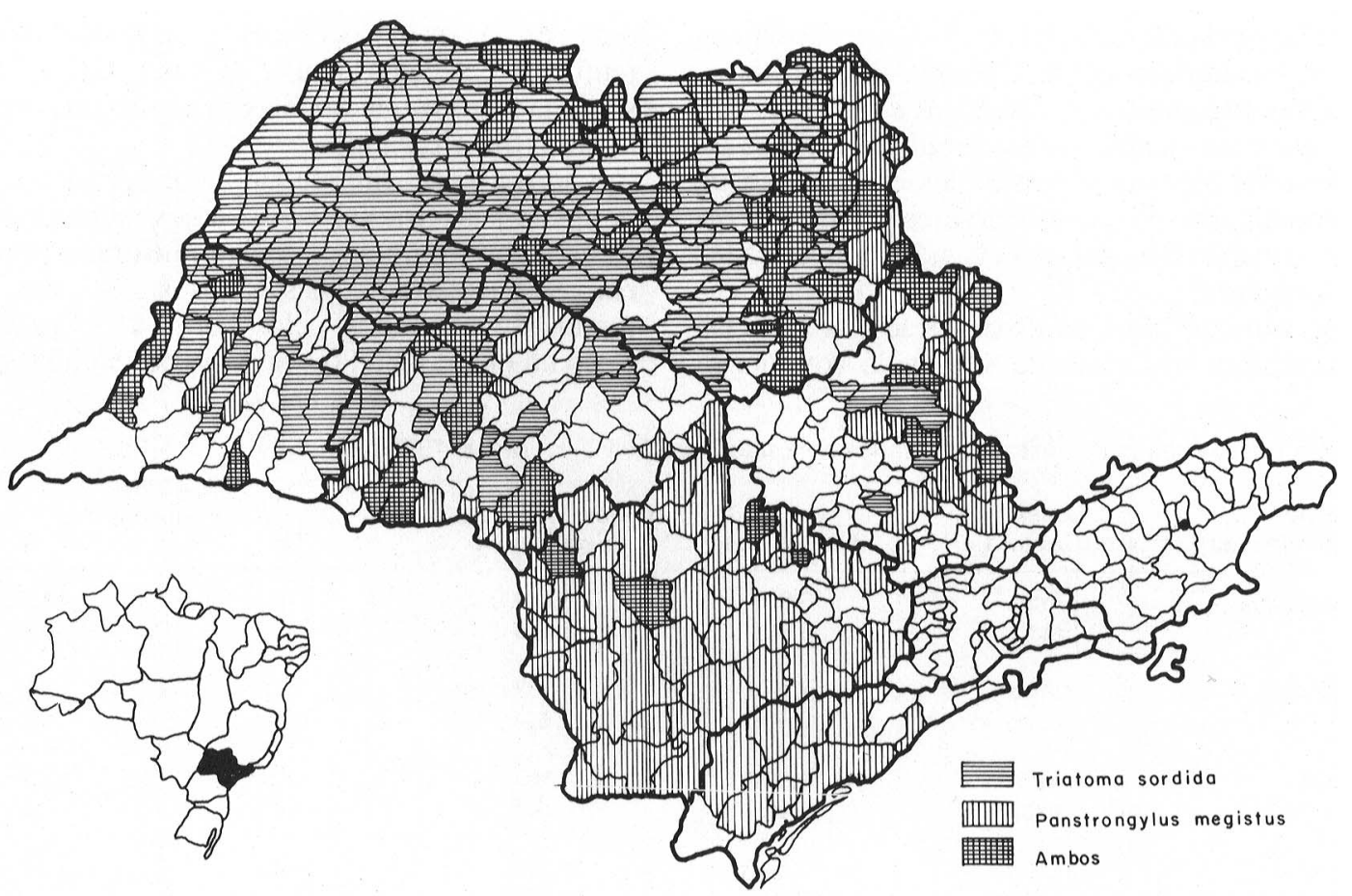

Fig. 1. Municipios com presença de Triatoma sordida e Panstrongylus megistus no Estado de São Paulo, Brasil, 1985 e 1986. 
No Estado de São Paulo, durante a fase mais ativa da campanha antitriatomínica, enquanto $T$. infestans cedia à alta pressão de inseticida, foi possível observar resposta pouco satisfatória de $T$. sordida e $P$. megistus aos métodos de controle ${ }^{1,9,10}$.

Informações referentes às espécies triatomínicas capturadas em 1985 e 1986 (Tabela 1) dão conta que $T$. sordida representa $79,8 \%$ dos exemplares, sendo que $77,4 \%$ destes estão presentes no ambiente peridomiciliar,com pequena parcela de indivíduos infectados $(0,8 \%)$. Esta espécie encontra-se bastante difundida no Estado, graças a sua valência ecológica que lhe permite freqüentar ecótopos variados, muitos destes com pequena probabilidade de alimento, e devido ao scu maior potencial reprodutivo com taxas de sobrevivência bastante elevadas e grande produção de adultos. Panstrongylus megistus está presente em 15,2\% das capturas, apresentando índices de infecção da ordem de $7,9 \%$, justificados pela sua associação alimentar com múltiplos vertebrados, domiciliados ou não, fato já bastante conhecido 2,10 .

Encontro de $T$. infestans tem sido bastante esporádico referindo-se 3 exemplares no período, sem infecção natural, introduzidos passivamente no Estado.

Embora na categoria "outros" estejam incluídas as presenças de Rhodnius neglectus, Triatoma arthurneivai, $P$. diasi, $P$. geniculatus, $R$. domesticuse T. tibiamaculata, estes dois últimos têm sido responsáveis pela elevada positividade verificada nas capturas do intradomicílio. Classificados por Barreto ${ }^{1}$ como triatomíneos silvestres, $R$. domesticus e $T$. tibiamaculata quando adultos invadem ecótopos artificiais. Os habitats silvestres desses triatomíneos são constituídos por ocos de árvores e ninhos de ratos e de marsupiais, daí seus elevados indices de infecção natural.

$\mathrm{Na}$ situação atual da distribuição das espécies triatomínicas no Estado de São Paulo constata-se que, a exemplo do que tem sido observado em outras áreas do País ${ }^{3}$, onde também se eliminou a espécie mais domiciliada ( $T$. infestans), o peridomicílio exerce papel importante na manutenção de focos de "barbeiros". Aí existem construções precárias que abrigam animais que fazem parte da cadeia alimentar de triatomíneos, constituindo-se ecótopo excelente para o estabelecimento de colônias 10 .

As espécies triatomínicas presentes no Estado atualmente são predominantemente peridomiciliárias e a invasão do intradomicnlio por insetos adultos tem sido frequiente, justificando-se uma atividade constante de estímulo à notificação junto à população, através de campanhas de conteúdo educativo e da pronta resposta da SUCEN.

O trabalho contínuo das equipes de campo durante anos seguidos "casa a casa" das localidades rurais teve um papel importante na conscientização do morador quanto ao significado da presença de insetos hematófagos em suas casas e, principalmente, na tomada de decisão em coletar esses insctos e encaminhá-los diretamente para a SUCEN, ou através do centro de saúde ou escola rural. Dados da Tabela 2 confirmam este fenômeno. Observa-se que $85,6 \%$ dos triatomíneos capturados dentro da casa estiveram condicionados à notificação, isto é, foram encaminhados pelo morador. Este aspecto tem um significado importante quanto ao risco de transmissão da doença de Chagas por estes insetos, uma vez que os índices de infeç̧ão natural de triatomíncos coletados dentro da casa $(6,6$ e $3,2 \%)$ são maiores do que aqueles presentes no peridomicílio $(0,9$ e $2,5 \%)$.

Outro aspecto importante a considerar é o fato de que a pesquisa nas casas, cujos moradores encaminharam "barbeiros" tem-se mostrado positiva em cerca de $26 \%$, e, nestes casos, $20 \%$ dos triatomíneos capturados pelas equipes de pesquisa estão no intradomicilio e $80 \%$ no peridomicílio.

Tabela 1. Espécies triatomínicas capturadas no Estado de São Paulo, Brasil, 1985 a 1986.

\begin{tabular}{|c|c|c|c|c|c|}
\hline $\begin{array}{l}\text { Espécies } \\
\text { Triatominicas }\end{array}$ & $\begin{array}{l}\text { Local de } \\
\text { Captura }\end{array}$ & $\begin{array}{c}\mathrm{Tr} \\
\text { Capturados }\end{array}$ & $\begin{array}{l}\text { eos } \\
\text { Examinados }\end{array}$ & $N^{Q}$ & $\%$ \\
\hline P. megistus & $\begin{array}{l}\text { intra } \\
\text { extra }\end{array}$ & $\begin{array}{l}3.057 \\
3.646\end{array}$ & $\begin{array}{l}2.446 \\
3.358\end{array}$ & $\begin{array}{l}278 \\
180\end{array}$ & $\begin{array}{r}11,4 \\
5,4\end{array}$ \\
\hline T. sordida & $\begin{array}{l}\text { intra } \\
\text { extra }\end{array}$ & $\begin{array}{c}7.937 \\
27.160\end{array}$ & $\begin{array}{c}6.587 \\
22.624\end{array}$ & $\begin{array}{r}53 \\
198\end{array}$ & $\begin{array}{l}0,8 \\
0,9\end{array}$ \\
\hline Outros & $\begin{array}{l}\text { intra } \\
\text { extra }\end{array}$ & $\begin{array}{r}1.546 \\
607\end{array}$ & $\begin{array}{r}1.153 \\
505\end{array}$ & $\begin{array}{l}43 \\
14\end{array}$ & $\begin{array}{l}3,7 \\
2,8\end{array}$ \\
\hline Sub total & $\begin{array}{l}\text { intra } \\
\text { extra }\end{array}$ & $\begin{array}{l}12.543 \\
31.413\end{array}$ & $\begin{array}{l}10.187 \\
26.487\end{array}$ & $\begin{array}{l}374 \\
392\end{array}$ & $\begin{array}{l}3,7 \\
1,1\end{array}$ \\
\hline Total & & 43.956 & 36.674 & 766 & 2,1 \\
\hline
\end{tabular}


Tabela 2. Captura de triatomíneos segundo atividade de rotina e notificaçăo. Estado de Săo Paulo, Brasil, 1985 a 1986.

\begin{tabular}{lccccc}
\hline \multirow{2}{*}{ Atividade } & Local de & \multicolumn{2}{c}{ Triatomineos } & Positivos & No \\
\hline Rotina & Captura & Capturados & Examinados & 96 & 6,6 \\
& intra & 1.810 & 1.462 & 162 & 0,9 \\
\hline & extra & 21.514 & 17.271 & 258 & 1,4 \\
\hline Notificaçåo & Sub total & 23.324 & 18.733 & 278 & 3,2 \\
& intra & 10.733 & 8.725 & 230 & 2,5 \\
\hline & extra & 9.899 & 9.216 & 508 & 2,8 \\
\hline Total & Sub total & 20.632 & 17.941 & 766 & 2,1 \\
\hline
\end{tabular}

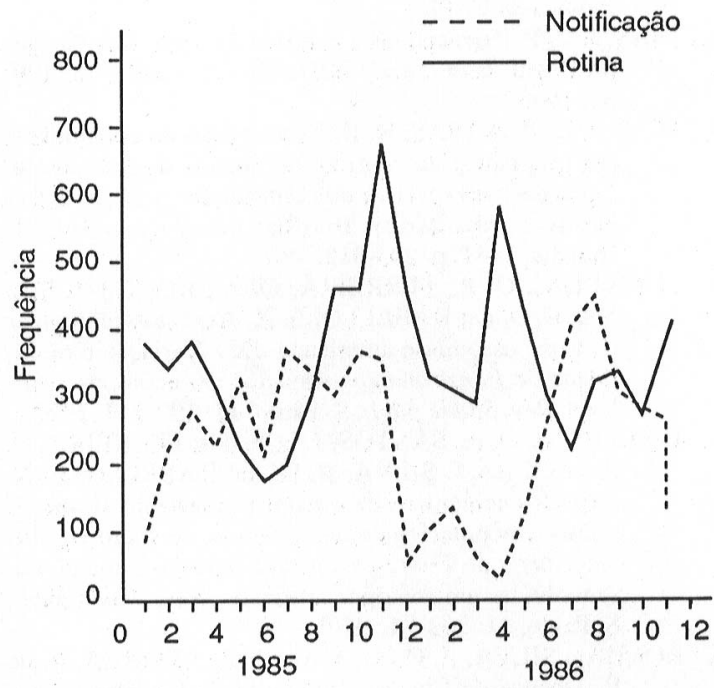

Fig. 2. Frequência mensal de casas positivas segundo rotina e notificação. Estado de São Paulo, Brasil, 1985 e 1986.

A estes achados são pertinentes algumas considerações:

- As equipes de campo, com longos anos de experiência e com grande domínio da pesquisa triatomínica, alcançam grande êxito na captura de triatomíneos no peridomicílio, local onde esses insetos mantêm suas colônias.

- A notificação de triatomíneos pela população é a melhor forma de se efetuar a vigilância no intradomicílio.

A distribuição mensal das casas em que o morador notifica o "barbeiro" e das casas onde as equipes de campo encontram triatomíneos em seu trabalho de rotina, avaliada na Figura 2, mostra que em 1985 ocorreram mais notificações no primeiro e nos dois últimos meses do ano. No período de fevereiro a outubro as diferenças entre rotina e notificação são menores, podendo não

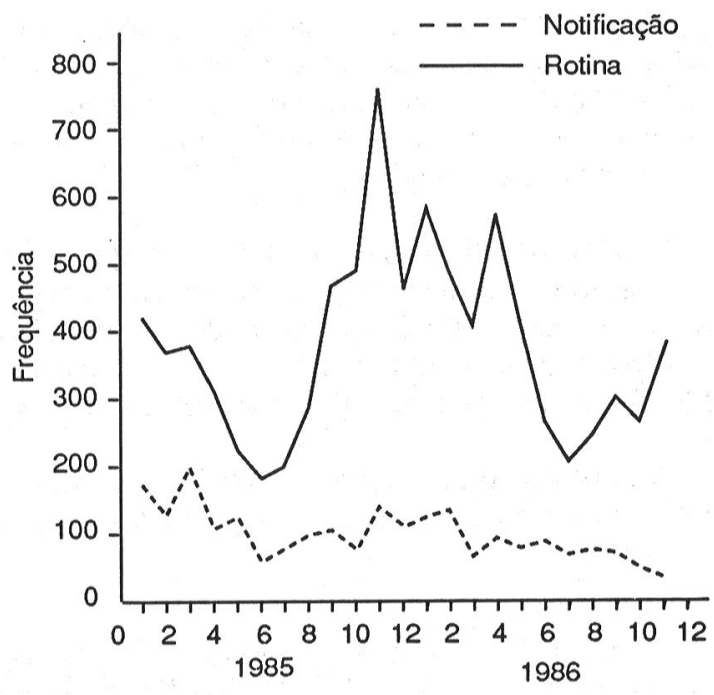

Fig. 3. Triatomíneos notificados capturados no intra $\theta$ no peridomicílio. Estado de São Paulo, Brasil, 1985 e 1986.

haver diferenças significantes entre elas. Já em 1986, estas diferenças procedem para os cinco primeiros e nos dois últimos meses do ano. Assim, ao serem considerados os dois anos, de outubro a maio, certamente ocorreram diferenças significativas entre rotina e notificação, com domínio marcante desta última. Neste período verifica-se maior atividade de dispersão de triatomíneos adultos que predomina no primeiro semestre do ano (Triatoma sordida) e no último trimestre do ano (Panstrongylus megistus).

Ainda confirmando pesquisas clássicas de Forattini e col. ${ }^{5,6}$ que referem maior atividade biológica de triatomineos nos meses mais quentes do ano com maior dispersão de formas adultas, a observação da Figura 3 mostra grande número de triatomíneos notificados, capturados pelo morador no intradomicílio, no período de setembro a maio. 
Tratam-se de exemplares fêmeas em sua grande maioria que, já fecundadas migram abandonando as colônias e assim, penetram nas casas com mais freqüência, podendo carrear consigo a infecção natural e o risco de transmissão.

\section{Conclusóes}

- No contexto atual da distribuição e do comportamento das espécies triatomínicas, vetoras da doença de Chagas no Estado de São Paulo, a vigilância entomológica deve ser pensada em caráter permanente e entendida como indispensável à manutenção dos domicílios livres desses insetos.

- O risco de colonização nas casas deve ser convenientemente avaliado, e estará sempre presente na medida em que não seja alterado o ambiente ou as condiçбes que favorecem a introdução e repovoamento dos triatomíneos.

- Na vigilância do intradomicílio a participação da população é de fundamental importância e deverá ser estimulada através de fluxo de informações constantes e de retroalimentação onde a questão educativa deve ser contemplada amplamente.

- A notificação de triatomíneos pela população é a melhor forma de se efetuar a vigilância no intradomicílio.

WANDERLEY, D. M. V. [Surveillance of Chagas' disease vectors in the State of S. Paulo, Brazil]. Rev. Saúde públ., S. Paulo, 25: 28-32, 1991. Drastic modifications in agrarian space, with the reductions of primary forests, along with changes in rural production systems have led to the growth of salaried employment and also caused rural workers to move to the peripheral areas of cities. There has also been an intense and continuous intervention undertaken by the Superintendência de Controle de Endemias (SUCEN) through the use of pesticides in the fight against triatomine bugs in houses and peridomestic areas of the State of S. Paulo. All these factors have contributed to the interruptions of the natural transmission of Chagas' disease in S. Paulo. The next step in the program was the integration of vertical vector control into primary heal th care involving community participation. The several years of SUCEN's work among the rural communities has encouraged them to notify the presence of triatominae bugs, and prompt response to each notification has led to the growth of the process of permanent participation. Data from 1985 and 1986 showed that $85.6 \%$ of the bugs captured inside houses were noti- fied by the population, which confirms that the best way to maintain the epidemiologic surveillance of Chagas' disease by the mobilization of local communities for effective participation in vector surveillance.

Keywords: Trypanosomiasis, South American, prevention and control. Population surveillance. Insect control. Consumer participation.

\section{Referências Bibliográficas}

1. BARRETO, M. P. Epidemiologia. In: Brener, Z. \& Andrade, Z., ed. Trypanosoma cruzi e doença de Chagas. Rio de Janeiro, Guanabara Koogan, 1979.p. 89-151.

2. BURALII, G. M. Estudo do controle dos triatomíneos domiciliados no Estado de São Paulo. São Paulo, 1985. [Dissertação de Mestrado-Faculdade de Saúde Pública da USP].

3. DIAS, J. C. P. Perspectivas e controle da doença de Chagas no Brasil. Cad. Saude públ., Rio de Janeiro, 2: 129. 33, 1986.

4. DIAS, J. C. P. \& DIAS, R. B. Participação da comunidade em programas de controle da doença de Chagas. In: Situação e perspectiva do controle das doenças infecciosas e parasitárias. Brasília, Ed. Universidade de Brasília, 1981. p. 293-312.

5. FORATTINI, O. P.; FERREIRA, O. A.; ROCHA E SILVA, E. O. da; RABELLO, E. X. Aspectos ecológicos da tripanossomíase americana. IX - Variação e mobilidade de Panstrongylus megistus em ecótopos anificiais. Rev. Saúde públ., S. Paulo, 11: 199-213, 1977.

6. FORATTINI, O. P.; SANTOS, J. L. F. dos; FERREIRA, O. A.; ROCHA E SILVA, E. O. da; RABELLO, E. X. Aspectos ecológicos da tripanossomíase americana. $X$ - Dados populacionais das colônias de Pantrongylus megistus e de Triatoma sordida espontaneamente desenvolvidas em ecótopos artificiais. Rev. Saúde públ., S. Paulo, 11: 362-74, 1979.

7. ROCHA e SILVA, E. O. da; MALUF, J.; CORREA, R, de $R$. Doença de Chagas: atividades de vigilância entomológica numa área do Estado de São Paulo, Brasil. Rev. Saúde públ., S. Paulo, 4: 129-45, 1970.

8. SILVA, L. J. Desbravamento, agricultura e doença: a doença de Chagas no Estado de São Paulo. Cad. Saúde públ., Rio de Janeiro, 2:124-40, 1986.

9. SOUZA, A. G.; WANDERLEY, D. M. V.; BURALLI, G. M.; ANDRADE, J. C. R. de. Consolidation of the control of Chagas' disease vectors in the State of São Paulo. Mem. Inst. Oswaldo Cruz, 79(supl.): 125-31, 1984.

10. WANDERLEY, D. M. V. Análise da atual estratégia do Programa de controle da doença de Chagas no Estado de São Paulo. São Paulo, 1987. [Dissentação de Mestrado - Faculdade Saúde Pública da USP].

11. WANDERLEY, D. M. V.; ANDRADE, J. C. R. de; MENEGUETTI, L. C.; CHINELATTO, M. J. Programa de controle da doença de Chagas no Estado de São Paulo; Relatório Anual: 1983. São Paulo, SUCEN, 1983. [Mimeografado]. Aprovado para publicaçāo em 25/10/1990 